\title{
An Ontology-driven Adaptive System for the Patient Treatment Management
}

\author{
Emna Mezghani \\ LAAS-CNRS, Université de Toulouse, CNRS, INSA, Toulouse, \\ France \\ Luxembourg Institute of Science and Technology, 5, Avenue des \\ Hauts-Fourneaux, L-4362, Esch/Alzette, Luxembourg
}

\author{
Marcos Da Silveira, Cédric Pruski \\ Luxembourg Institute of Science and Technology, 5, Avenue des \\ Hauts-Fourneaux, L-4362, Esch/Alzette, Luxembourg
}

\author{
Ernesto Exposito and Khalil Drira \\ LAAS-CNRS, Université de Toulouse, CNRS, INSA, Toulouse, France
}

\begin{abstract}
Advances in the Web and healthcare data capture technologies have far-reaching benefits for the development of new clinical decision support systems that accelerate decisionmaking and generate personalized treatments. However, the diversity of healthcare data formats, the lack of computer interpretable representation of medical interventions, and the distribution of reliable medical knowledge sources constitute important barriers to better support the medical decision process. To deal with these issues, we propose the Treatment Plan Ontology (TPO) that formalizes medical interventions, and allows medical systems sharing and reasoning over them. This knowledge together with the acquired patient data are then reused by the autonomic processes that we have developed in order to timely detect anomalies and support the physicians in personalizing the patient treatment at the right time. We demonstrate the system efficiency through a use case for managing hyperglycemia in type 2 diabetes.
\end{abstract}

Keywords- Treatment Adaptation; Autonomic Computing; Semantic Web; Knowledge Representation

\section{INTRODUCTION}

Advances in healthcare technologies including mobile systems, medical sensors and wearable computing foster the generation of real time data. Leveraging these technologies to automate the integration of the patient data with the medical knowledge paves the way for developing smart clinical decision support systems (CDSS).

The efforts of Medical and Informatics communities to formalize medical knowledge from clinical guideline resulted into several computer-interpretable guidelines (CIGs) described in different languages to elucidate and facilitate the decision process [1]. Recently, considerable efforts have been invested to bring the medical knowledge onto the Web using Semantic Web technologies [2] in order to integrate different sources, to update, to share and reuse this knowledge. Noticeably, drugdrug interactions, drug-food interactions and adverse drug reactions are being published and accessible as linked data [3, 4]. Integrating these sources with the medical interventions described by medical experts is crucial for more fine-grained decisions. Nevertheless, there is a lack of flexible representation of medical interventions that underpin the treatment adaptation and the dynamic patient' context changes. Moreover, the acquisition of such knowledge is challenging since it requires a minimum of IT expertise to annotate the medical interventions and update decision rules.

Consequently, we proposed an ontology-driven adaptive system that provides a smart management of the patient treatment. We defined a Treatment Plan Ontology (TPO) that will be used for annotating medical intervention and their associated rules. Semantic Web technologies were adopted to develop a collaborative user-friendly environment that can hide the complexity of the formalism used by the system. Moreover, we developed reusable autonomic processes that exploit the annotated knowledge to automatically detect anomalies and assist the physicians in taking the right decision at the right time.

The remainder of this paper is organized as follows. Section 2 details the proposed adaptive system and delineates the Treatment Plan Ontology. Section 3 highlights the efficiency of our adaptive system through a diabetes use case. Section 4 discusses existing work dealing with knowledge-based systems for treatment adaptation. Finally, Section 5 concludes this paper and gives some directions for future work.

\section{PATIENT TREATMENT AdAPtATION}

In this section, we present an overview of our adaptive system and the Treatment Plan Ontology which is the foremost element for automating the decision process.

\section{A. An Overview of Treatment Adaptation}

We adopted the autonomic computing paradigm [5] to adapt and manage the patient's treatment [6] in order to accelerate the decision-making and avoid the patient health complications. Originally inspired from the human autonomic nervous system, the autonomic computing has been proposed to enable self-managed properties through implementing the $M A P E-K$ pattern (Monitoring, Analysis, Plan, Execution and Knowledge).

In our work, the Monitoring process is responsible of collecting patient data coming from medical sensors and wearable devices, and/or measured by health professionals. Once the data is collected, the Analysis process is triggered to identify patient health deterioration. It may implement detection rules, or predictive algorithms such as machine learning. According to the severity of the detected (or predicted) anomaly, an alert will be sent to the Plan process 
that automatically searches and filters the appropriate treatments based on the patient medical conditions, the interventions contraindications, the medical interactions and the drug side effects. Finally, the personalized recommendations are sent to the appropriate physician for approval or modification. The aforementioned processes operate on the Knowledge which is the fundamental element that enables the coordination of the MAPE processes for the smart management of the patient treatment.

In this paper, we delineate the knowledge component and we propose the Treatment Plan Ontology (TPO) which semantically formalizes the medical interventions including their conditions and side effects, and represents some concepts pertaining to the patient context in order to assist the physicians in making personalized decisions that meet the patient profile and preferences.

\section{B. Treatment Plan Ontology}

We followed a collaborative methodology [7] for formalizing the medical knowledge, based on discussions with medical experts. The output is a flexible TPO schema that builds the bridge between the artificial intelligence planning and the semantic representation of medical knowledge in order to grasp the computational intelligence. Our TPO semantically represents the medical interventions as actions characterized through preconditions and side effects in order to reach the patient objective. The main objective is to provide a generic model, which is the basis for reasoning, to automate the decision making according to the identified problems.

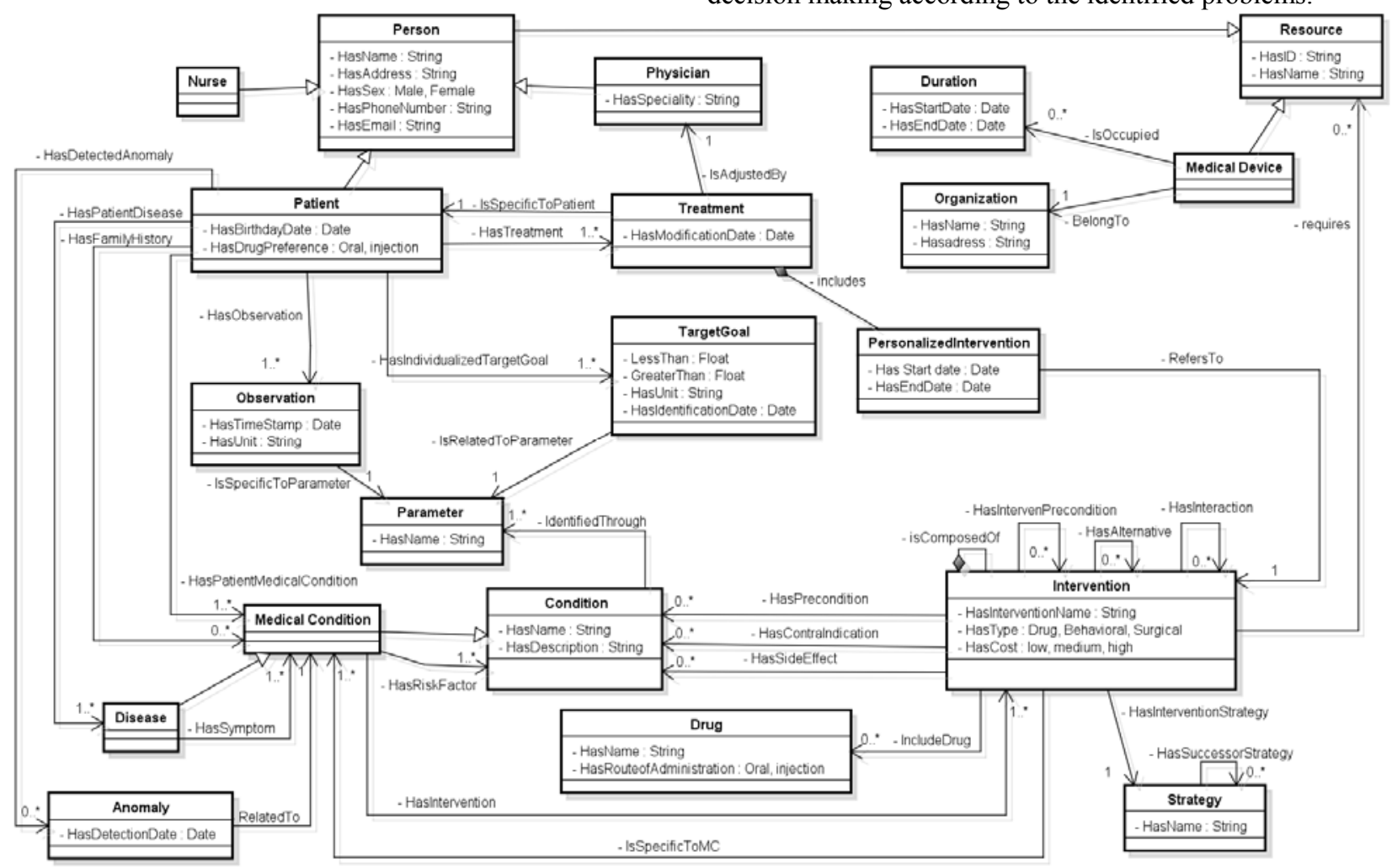

Figure 1. Depiction of the main classes and relationships with their cardinalities in the proposed Treatment Plan Ontology

Figure 1 portrays the main classes and relationships of our TPO that represents a formalization of a problem-solving system for chronic disease management. We introduced in TPO the medical condition class which is defined by the Segen's Medical Dictionary as "A disease, illness or injury; any physiologic, mental or psychological condition or disorder... A biological or psychological state which is within the range of normal human variation is not a medical condition". Each condition is identified through measurable parameters, for instance the HbAlc identifies a high or low blood sugar medical condition. We consider that a disease has symptoms which are also medical conditions. Idem, each medical condition has risk factors which are defined as conditions that could make a person more likely to develop a disease or to amplify the symptoms of an existing disease. For example, both obesity and hypertension are risk factors of the type 2 diabetes. These medical conditions can be minimized and/or stabilized through medical interventions that help achieving the patient goal. An intervention can be behavioral, surgical or drug-based (characterized by its route of administration). To automate the selection of a treatment, our TPO associates each medical intervention with the appropriate disease strategy, and encodes the right strategy selection through the HasSuccessorStrategy property. For example, in type 2 diabetes, four strategies are identified: the "monotherapy", the "dual combination", the "triple combination" and the "complex insulin" [8]. If a patient who is treated with a "monotherapy" strategy presents an increased blood sugar, it is recommended to prescribe a "dual combination" strategy. Moreover, TPO describes the medical contraindication as well as side effects expressed as conditions. 
Focusing on treatment personalization, TPO includes concepts related to the patient profile such as diseases, medical conditions including the allergies, the monitored signs, the family history, the current treatments and preferences. Likewise, to enable the dynamic adaptation, our TPO introduces the TargetGoal class which is specific to the patient. This goal is fixed by the physician and strongly depends on the patient medical conditions and disease severity stage. It will be used by the Analysis process to detect anomalies. Each anomaly is associated to a medical condition in order to be explored by the Plan process to extract the interventions that address the detected anomaly. TPO has been conceived to allow the medical experts populating the decision rules through creating relations among TPO instances. Thus, decision rules can be easily updated by experts and reused by the autonomic processes to detect anomalies and generate personalized recommendations. This fruitful model, implemented in OWL [9], can be extended and instantiated for other chronic disease.

To hide the complexity of annotating the medical interventions, we developed a collaborative user-friendly platform that allows medical experts sharing medical knowledge. Our collaborative platform is based on Semantic MediaWiki ${ }^{1}$ (SMW). SMW unleashes the power of wikis for collaborative knowledge management, and ontologies for providing a common understanding of the domain. It offers mapping mechanisms for formalizing annotations embedded in wiki pages into OWL DL ontology language [10]. We installed around 30 extensions to guarantee an easy interaction with the experts, and to provide visualization services. Our Semantic Web platform also integrates OpenLDAP ${ }^{2}$ to control the access to the patient data. We extended our platform with Fuseki ${ }^{3}$, a RDF store in order to provide a SPARQL endpoint that allows the external autonomic processes automatically seek the appropriate information. The elaborated SPARQL queries are generic and parameterized. They do not integrate hard-coded conditions related to the patient or to the disease, which makes the autonomic processes reusable.

\section{USE CASE: DIABETES MANAGEMENT}

In this section, we demonstrate the efficiency of our adaptive system through the ability of the autonomic processes to retrieve the appropriate information from TPO in order to automatically detect the patient medical anomalies, and select personalized recommendations when managing the hyperglycemia in type 2 diabetes. The first step consists in using our collaborative platform for annotating the medical interventions and strategies published in the American Diabetes Association (ADA) and European Association for the Study of Diabetes (EASD) [8].

\section{A. Use Case Description}

We referred to a case study published in the Clinical Diabetes journal [11] in order to simulate our system, and compare the recommendations that our system proposes to what the use case claims. The use case describes an elderly patient who is having type 2 diabetes for 11 years. He has

\footnotetext{
${ }^{1}$ Semantic MediaWiki : https://www.semantic-mediawiki.org/ ${ }^{2}$ OpenLDAP: http://www.openldap.org/

${ }^{3}$ Fuseki: https://jena.apache.org/documentation/serving data/
}

obesity, and his medical history includes hypertension treated with lisinopril, hyperlipidemia treated with pravastatin, rightknee osteoarthritis, a right hip replacement at the age of 61 years, pneumothorax at the age of 35 years, and benign prostatic hypertrophy. He doesn't present any complication from his diabetes, and prefers oral drug than injection. As a first treatment, the physician prescribed for him metformin (1000 mg twice daily). According to the use case description [11], the patient HbAlc target goal is fixed to $7 \%$.

\section{B. Simulation of the Adaptation Process}

We registered the patient having the aforementioned profile in our platform. We deployed a MAPE loop that simulates the dynamic adaptation based on the patient context changes. The Monitoring process periodically measures the patient HbAlc parameter. The frequency of the monitoring is configured according to the patient medical conditions and to the measured parameter. The Analysis process retrieves the patient TargetGoal from the populated knowledge (in our case it is equal to $7 \%$ ). If the monitored data is greater than the last measurement and greater than the patient target goal, the Analysis process detects an anomaly. This anomaly is associated to the high blood sugar medical condition, and is automatically sent to the Plan process. In this paper, the Plan process operates on the medical knowledge annotated based on TPO and generates the right recommendations based on the disease risk factors, the medical interactions and side effects, the patient profile and preference as the main criteria for the treatment personalization. Figure 2 illustrates an excerpt of the MAPE loop enactment. Our system is able to argue its selection in order to assist the physicians.

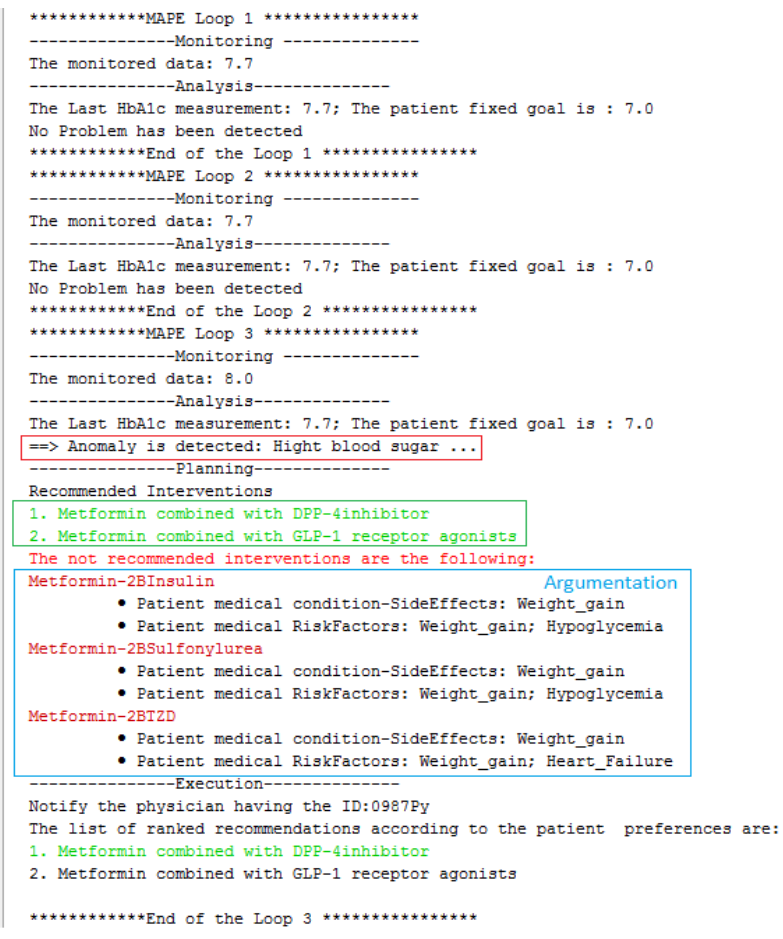

Figure 2. An excerpt of the autonomic processes enactment

According to the use case description [11], the patient has obesity and hypertension history, interventions that include side 
effects or risk factors such as weight gain and heart failure should be avoided. Moreover, the patient is at an early stage of the hyperglycemia, interventions that may cause hypoglycemia such as the insulin and sulfonylurea should be avoided. The Plan process retains two recommendations, (1) metformin combined with DPP-4-inhibitor (oral drugs) and (2) metformin combined with GLP-1 receptor agonists (oral+injection), which are ranked according to the patient preference (oral). The ranked list is sent to the appropriate physician to validate or modify the treatment, and contact his patient.

\section{RELATED WORK}

Many research activities investigated the medical knowledge formalization based on Semantic Web for the development of CDSS [12]. However, few studies focus on the dynamic adaptation of the patient treatment based on the context changes, which requires more flexible representation of the medical knowledge. Alexandrou et al. [13] proposed an Adaptive Clinical Pathway Ontology for adapting the patient clinical workflow. The ontology is implemented in OWL and the adaptation process is based on SWRL rules, while the knowledge acquisition is done by experts through Protégé. Similarly, Yao and Kumar [14] proposed to use Protégé to acquire the clinical context ontology and SWRL rules to generate recommendations based on the patient context changes. Their work support the patient treatment generation and prescription checking based on rules. However, these rules are static and hard-coded. In this context, we proposed the TPO schema that overcomes these problems and allows the autonomic processes automatically exploring the annotated knowledge based on flexible SPARQL queries and supporting the integration of external linked data such as DrugBank. Moreover, we developed a collaborative user-friendly interface that interacts with the medical experts to semantically annotate the medical interventions based on TPO.

Lasierra et al. [15] presented an interesting work that relies on autonomic computing to manage patients with chronic disease. The authors used ontology to enable semantic interoperability when monitoring the patient at home using medical sensors. However, their work is interested in the monitoring and analysis processes in order to identify alarms and send warnings to physicians and patients. Both Alexandrou et al. [13] and Lasierra et al. [15] did not consider encoding the characteristics of the medical interventions, which are the basis for the decision support and treatment personalization. Our proposed adaptive system aims at accelerating the decision making and continuously supervising the patient to timely take the right decision. It includes a semantic formalization of the medical interventions based on TPO, which plays the role of a mediator between medical experts and the autonomic processes.

\section{CONCLUSION}

We proposed an ontology-driven adaptive system for the smart management of the patient treatment based on autonomic computing. The adaptation is based on TPO which is a flexible semantic representation of the medical interventions and their characteristics that underpin the personalization of the patient treatment. To accelerate the decision-making and avoid health complications, we developed also a set of autonomic processes that rely on TPO to automatically detect medical problems and generate the appropriate recommendations at the right time. As proof of concept, we demonstrated the efficiency of our adaptive system through managing hyperglycemia scenario.

Currently, we are working on an advanced planning algorithm that integrates TPO with existing large scale knowledge sources such as DrugBank. Furthermore, we aim at evaluating the quality of our system through simulating multiple use cases based on experts' collaboration.

\section{ACKNOWLEDGMENT}

This research is entirely funded by the National Research Fund (FNR) of Luxembourg under the AFR project.

\section{REFERENCES}

[1] P. A. De Clercq, J. A. Blom, H. H. Korsten and A. Hasman. "Approaches for creating computer-interpretable guidelines that facilitate decision support". Artificial intelligence in medicine, vol. 31, 2004,pp 1-27.

[2] T. Berners-Lee, J. Hendler and O. Lassila. "The semantic web". Scientific american, vol. 284, 2001, pp 28-37.

[3] M. Samwald, A. Jentzsch, C. Bouton, C. S. Kallesøe, E. Willighagen, J. Hajagos, M. S. Marshall, E. Prud'hommeaux, O. Hassanzadeh and E. Pichler. "Linked open drug data for pharmaceutical research and development". Journal of cheminformatics, vol. 3, 2011, pp19

[4] A. Callahan, J. Cruz-Toledo, and M. Dumontier. "Ontology-based querying with Bio2RDF's linked open data". Journal of biomedical semantics, 2013, 4(Suppl 1), S1.

[5] J. O. Kephart and D. M. Chess. "The vision of autonomic computing". Computer, vol. 36, 2003, pp 41-50.

[6] E. Mezghani, M. Da Silveira, C. Pruski, E. Exposito, and K. Drira. "A perspective of adaptation in healthcare". Studies in health technology and informatics, In : MIE. 2014, pp 206-210.

[7] E. Mezghani, E. Exposito, and K. Drira. "A collaborative methodology for tacit knowledge management: Application to scientific research". Future Generation Computer Systems, vol. 54, 2016, pp 450-455.

[8] S. E. Inzucchi, R. M. Bergenstal, J. B. Buse, M. Diamant, E. Ferrannini, M. Nauck, A. L. Peters, A. Tsapas, R. Wender and D. R. Matthews. "Management of hyperglycemia in type 2 diabetes: a patient-centered approach position statement of the American Diabetes Association (ADA) and the European Association for the Study of Diabetes (EASD)". Diabetes care, vol.35, 2012, pp1364-1379.

[9] Treatment Plan Ontology. http://homepages.laas.fr/emezghan/TPO.owl.

[10] M. Krötzsch, D. Vrandečić and M. Völkel. "Semantic mediawiki”. In : The Semantic Web-ISWC 2006. Springer Berlin Heidelberg, p. 935-942.

[11] S. W. Lahiri. "Management of type 2 diabetes: what is the next step after metformin?" Clinical Diabetes, vol. 30, 2012, pp 72-75.

[12] D. Riaño, F. Real, J. A. López-Vallverdú, F. Campana, S. Ercolani, P. Mecocci, R. Annicchiarico and C. Caltagirone. "An ontology-based personalization of health-care knowledge to support clinical decisions for chronically ill patients". Journal of biomedical informatics, vol. 45, 2012, pp 429-446.

[13] D. A. Alexandrou, I. E. Skitsas and G. N. Mentzas. "A holistic environment for the design and execution of self-adaptive clinical pathways". Information Technology in Biomedicine, IEEE Transactions on, vol. 15, 2011, pp 108-118.

[14] W. Yao and A. Kumar. "CONFlexFlow: Integrating flexible clinical pathways into clinical decision support systems using context and rules". Decision Support Systems, vol. 55, 2013, pp 499-515.

[15] N. Lasierra, A. Alesanco, S. Guillén and J. Garcia. "A three stage ontology-driven solution to provide personalized care to chronic patients at home". Journal of biomedical informatics, vol. 46, 2013, pp 516-52 